\title{
Encounters with Rheumatologists in a Publicly Funded Canadian Healthcare System: A Population-based Study
}

\author{
Jessica Widdifield(D), Sasha Bernatsky (1D, Janet E. Pope (i), Vandana Ahluwalia (iD), \\ Claire E.H. Barber ${ }^{(}$, Lihi Eder ${ }^{(1)}$, Bindee Kuriya ${ }^{\circledR}$, Vicki Ling ${ }^{(1)}$, J. Michael Paterson (1), \\ and J. Carter Thorne ${ }^{(1)}$
}

ABSTRACT. Objective. To quantify population-level and practice-level encounters with rheumatologists over time.
Methods. We conducted a population-based study from 2000 to 2015 in Ontario, Canada, where all
residents are covered by a single-payer healthcare system. Annual total number of unique patients
seen by rheumatologists, the number of new patients seen, and total number of encounters with
rheumatologists were identified.
Results. From 2000 to 2015 , the percentage of the population seen by rheumatologists was constant
over time $(2.7 \%)$. During this time, Ontario had a stable supply of rheumatologists $(0.8$ full-time
equivalents/75,000). From 2000 to 2015 , the number of annual rheumatology encounters increased
from 561,452 to 786,061 , but the adjusted encounter rates remained stable over time (at 62 encounters
per 1000 population). New patient assessment rates declined over time from 10 new outpatient assess-
ments per 1000 in 2000 to 6 per 1000 in 2015 . The crude volume of new patients seen annually
decreased and an increasing proportion of rheumatology encounters were with established patients.
We observed a shift in patient case mix over time, with more assessments for systemic inflammatory
conditions. Rheumatologists' practice volumes, practice sizes, and the annual number of days
providing clinical care decreased over time.
Conclusion. Over a 15 -year period, the annual percentage of the population seen by a rheumatologist
remained constant and the volume of new patients decreased, while followup patient encounters
increased. Patient encounters per rheumatologist decreased over time. Our findings provide novel
information for rheumatology workforce planning. Factors affecting clinical activity warrant further
research. (First Release November $152019 ; \mathrm{J}$ Rheumatol $2020 ; 47: 468-76$; doi:10.3899/jrheum.190034)

Key Indexing Terms:

RHEUMATOLOGY

WORKFORCE

\author{
CLINICAL ACTIVITY \\ HEALTH SERVICES NEEDS AND DEMAND
}

\begin{abstract}
From Sunnybrook Research Institute, Holland Bone and Joint Program; University of Toronto, Institute of Health Policy, Management and Evaluation; ICES; Arthritis Research Canada; Women's College Research Institute; Sinai Health System, University of Toronto, Toronto, Ontario; McGill University, Department of Epidemiology; Research Institute of the McGill University Health Centre, Montreal, Quebec; William Osler Health System, Brampton, Ontario; The Cumming School of Medicine, University of Calgary, Calgary, Alberta; McMaster University, Department of Family Medicine, Hamilton; Western University, Schulich School of Medicine \& Dentistry; St. Joseph's Health Care London, London, Ontario; Southlake Regional Health Centre, Newmarket, Ontario, Canada.

This study was funded by an operating grant from the Canadian Initiative for Outcomes in Rheumatology Care, which played no role in the design or conduct of the study other than providing peer review of the study proposal. This study was supported by ICES (www.ices.on.ca), which is funded by an annual grant from the Ontario Ministry of Health and Long-Term Care (MOHLTC). Parts of this material are based on data and/or information compiled and provided by the Canadian Institute for Health Information (CIHI). However, the analyses, conclusions, opinions, and statements expressed in the material are those of the authors and not necessarily those of $\mathrm{CIHI}$, and are independent from the funding sources. No endorsement by ICES or the Ontario MOHLTC is intended or should be inferred. SB holds a career award from the Fonds de la recherche en santé du Québec.
\end{abstract}

\author{
J. Widdifield, PhD, Sunnybrook Research Institute, Holland Bone and \\ Joint Program, and University of Toronto, Institute of Health Policy, \\ Management and Evaluation, and ICES; S. Bernatsky, MD, FRCPC, PhD, \\ McGill University, Department of Epidemiology, and Research Institute of \\ the McGill University Health Centre; J.E. Pope, MD, FRCPC, MPH, \\ Western University, Schulich School of Medicine \& Dentistry, and St. \\ Joseph's Health Care London; V. Ahluwalia, MD, FRCPC, William Osler \\ Health System; C.E. Barber, MD, FRCPC, PhD, The Cumming School of \\ Medicine, University of Calgary, and Arthritis Research Canada; L. Eder, \\ MD, PhD, University of Toronto, Institute of Health Policy, Management \\ and Evaluation, and Women's College Research Institute; B. Kuriya, MD, \\ FRCPC, SM, Sinai Health System, University of Toronto; V. Ling, MSc, \\ ICES; J.M. Paterson, MSc, University of Toronto, Institute of Health \\ Policy, Management and Evaluation, and ICES, and McMaster University, \\ Department of Family Medicine; J.C. Thorne, MD, FRCPC, Southlake \\ Regional Health Centre. \\ Address correspondence to Dr. J. Widdifield, Holland Bone and Joint \\ Research Program, Sunnybrook Research Institute, 2075 Bayview \\ Avenue MG 352, Toronto, Ontario M4N 3M5, Canada. \\ E-mail: jessica.widdifield@utoronto.ca \\ Full Release Article. For details see Reprints and Permissions at \\ jrheum.org \\ Accepted for publication June 7, 2019.
}

Personal non-commercial use only. The Journal of Rheumatology Copyright (c) 2020. All rights reserved. 
In Canada and throughout the world, rheumatic and musculoskeletal diseases (RMD) are some of the most prevalent conditions affecting population health ${ }^{1}$. The aging demographic, increasing prevalence of RMD, and complexity of care management involved with comorbid illnesses in aging populations is expected to place greater demands on rheumatology services ${ }^{2}$.

Rheumatology workforces are increasingly challenged by too few physicians $3,4,5,6$. Physician surveys have identified major demographic changes of rheumatology workforces including baby boomer retirements, a millennial predominance, and an increase in female and part-time providers ${ }^{3,4}$. These changes, coupled with an increased demand for adult rheumatology care due to the growing and aging population and the rise of the burden of RMD, are likely to hinder clinical activity and reduce the supply of rheumatology services.

In Canada, rheumatologists are currently among the most frequent nonsurgical specialty referrals ${ }^{7}$ and they also have among the longest specialist wait times $8,9,10,11$. A better understanding of longitudinal trends in rates of rheumatology encounters with new and existing patients is needed to help identify why patients have such long waits for rheumatology care.

To better document the issues facing rheumatology care, more detailed information is needed regarding populations accessing rheumatology services and the clinical activity of the workforce in a population-based sample. Thus, we sought to describe population-level trends in patient encounters with rheumatologists between 2000 and 2015 in Ontario, Canada. In addition, we assessed trends in practice-level clinical activity of rheumatologists over time.

\section{MATERIALS AND METHODS}

Setting. Ontario is a large, diverse, multicultural province that constitutes about $40 \%$ of Canada's population, with a population of 11 million adults in $2015^{12,13}$. About $40 \%$ of the Canadian rheumatology workforce resides in Ontario ${ }^{4}$. All residents are covered by a universal, single-payer, public health insurance that includes hospital care and physicians' services, and in which access to rheumatologists requires referral from a physician or nurse practitioner.

Study design. We conducted a retrospective study using population-based health administrative databases in Ontario from 2000 to 2015.

Data sources. Annual population denominators (all residents 18 yrs and older) were ascertained from the Primary Care Population Database derived from the Ontario Health Insurance Plan (OHIP) Registered Persons Database, which includes all OHIP beneficiaries alive and who have accessed the healthcare system. Residents are eligible for OHIP if they are Canadian citizens, permanent residents (formerly called landed immigrants), or refugees who make their permanent and principal home in Ontario and are physically present in Ontario 153 days in any 12-month period. Physicians are reimbursed by submitting claims to OHIP for medical services provided.

We identified all patients 18 years and older with rheumatology encounters within the OHIP Claims History Database, which provides diagnoses, dates, and types of services associated with each rheumatology encounter. One diagnosis is provided with each claim, which represents the main "reason for the visit." These diagnoses are coded in a modification of the 8th revision of the International Classification of Diseases. Claims also include fee codes reflecting the types of services provided, and where the service was rendered (inpatient or outpatient setting) ${ }^{14}$.

Rheumatologists were identified using the ICES Physician Database, which is constructed and routinely validated using the OHIP Corporate Provider Database, the Ontario Physician Human Resources Data Centre Database, and physicians' OHIP billings. We included only active rheumatologists, defined as those with fee-for-service claims during each year (excluding pediatric rheumatologists).

These datasets are linked using unique, encoded patient and physician identifiers and are securely held and analyzed at ICES (www.ices.on.ca). ICES is a prescribed entity under section 45 of Ontario's Personal Health Information Protection Act. The use of data in this study was authorized under section 45 of Ontario's Personal Health Information Protection Act, which does not require review by a research ethics board.

Population-level measures. Contacts with rheumatologists were separately defined as any type of patient encounter (including inpatient, outpatient assessments, and other interactions which may include non-face-to-face encounters such as telemedicine, or review of laboratory test reports or bone densitometry reports), and outpatient assessments only (reflecting only face-to-face visits). Multiple fee codes billed by the same physician on the same patient on the same day were counted as 1 encounter (such as performing a consult and injecting a joint). We separately identified the annual total number of unique patients with at least 1 rheumatology encounter (including both inpatient and outpatient), only outpatient assessments, as well as the total number of rheumatology encounters and outpatient assessments (because patients may have multiple contacts throughout the year). Using annual population denominators, we determined the percentage of Ontario residents with encounters and assessments annually, as well as rates of total encounters and assessments expressed per 1000 population.

To identify new patients seen by rheumatologists each year, we applied a 3-year washout period ensuring patients had no prior rheumatology contacts. Annual new patient encounter and assessment rates, as well the percentage of new patients out of all rheumatology patients, and out of the total volume of rheumatology contacts were also stratified by any type of patient encounter and outpatient assessments only.

Diagnosis codes assigned at each outpatient assessment were used to assess for changes in the case mix of patients under outpatient rheumatology care over time. Among annual total outpatient assessments, we assessed the frequency of diagnosis codes for osteoarthritis (OA), rheumatoid arthritis (RA), ankylosing spondylitis, other inflammatory arthritis (such as seronegative or psoriatic arthritis), gout, connective tissue diseases (such as systemic lupus erythematosus, systemic sclerosis), other systemic autoimmune rheumatic diseases (such as polymyalgia rheumatica, vasculitis, sarcoidosis), regional musculoskeletal (MSK) conditions, osteoporosis, bone and spinal conditions, trauma and related conditions/injuries, and other conditions with diagnosis codes not defined in Supplementary Table 1 (which represent non-MSK-related conditions such as hypertension, infection; available with the online version of this article). Using the yearly population denominators, we assessed crude and age-and-sex standardized rates (directly standardized to the 2000 population)

Practice-level measures. We assessed the annual number, and clinical activity, of all active rheumatologists overall, and by clinical full-time equivalent (FTE) classification. Using annual fee-for-service billing claims, rheumatologists below the 40th percentile of total billings each year were classified as providing less clinical activity $(<1$ FTE); rheumatologists within the 40-60th percentile were classified as $1 \mathrm{FTE}$; and $>60$ th percentile as $>1 \mathrm{FTE}^{15}$. Because this FTE measure does not take into account the annual total number of days providing clinical care, we also determined the number of days with patient encounters and patient assessments per year. This alternative FTE measure identifies the number of rheumatologists who worked on at least 209 days out of the year [ 365 days in the year - 102 days (weekends) - 12 days (statutory holidays) - 30 days (vacations) - 9 days (attending conferences/continuing medical education [CME] activities/ meetings) $=209$ days .

Personal non-commercial use only. The Journal of Rheumatology Copyright $\subset$ 2020 . All rights reserved. 
Annual practice volumes (defined as the median no. patient encounters per yr) and practice sizes (defined as the median no. unique patients seen per yr) were assessed according to clinical FTE classification. Because rheumatologists may enter the workforce, die, move out of the province, retire, or take leaves of absence throughout the study period, rheumatologists were removed from the annual denominator of active rheumatologists if any physician had no claim for 365 days and their last claim date was assigned the date they exited the workforce. Rheumatologists were permitted to re-enter the workforce if they subsequently became active.

All analyses were performed using linked, encoded data held at ICES using SAS, version 9.2 (SAS Institute Inc.).

\section{RESULTS}

Population-level contacts with rheumatologists. Despite an increase in the population of Ontario during the study period, the percentage of the population seen by a rheumatologist annually remained constant over time at $2.7 \%$ (Table 1 ). The percentage of patients seen by a rheumatologist for an outpatient assessment also remained fairly constant (2\%).

The total number of rheumatology encounters increased from 561,452 annual patient encounters (382,074 outpatient assessments) in 2000 to 786,061 annual patient encounters (500,831 outpatient assessments) in 2015 (Table 1). The number of new patients seen annually decreased over time as well as the percentage of new patients out of the total patients under rheumatology care.

A breakdown of the types of rheumatology encounters is provided in Supplementary Figure 1 (available with the online version of this article), illustrating that the volume of inpatient encounters declined over time, whereas the volume of other patient interactions (e.g., non-face-to-face encounters such as laboratory review) increased over time.

From 2000 to 2015 , the total crude patient encounter rate increased $15 \%$ from 61.7 to 70.8 encounters per 1000 population (Figure 1). The annual total outpatient assessment rate was fairly constant over time (42-45 assessments per 1000). New patient encounter rates declined over time $(9.8$ new patient assessments per 1000 in 2000 to 6.2 new patient assessments per 1000 in 2015).

We observed a shift in the diagnostic case mix of patients over time, with rheumatologists seeing more systemic inflammatory conditions and a declining proportion of patients with regional MSK conditions (Table 2). The volume of RA encounters increased the most, with $21 \%$ of all assessments related to RA in 2000 versus $27 \%$ in 2015 . Crude patient assessment rates for systemic inflammatory conditions significantly increased over time and encounter rates for nonsystemic conditions (OA, regional MSK conditions) decreased (Supplementary Table 2, available with the online version of this article).

The standardized rates for age and sex for total encounters and outpatient assessments were fairly stable across years (Supplementary Table 3, available with the online version of this article). Adjusted rates for systemic inflammatory condi-

Table 1. Annual number of patients seen by rheumatologists and total patient encounters with Ontario rheumatologists between 2000 and 2015.

\begin{tabular}{|c|c|c|c|c|c|c|c|c|c|c|c|}
\hline \multirow[b]{2}{*}{ Year } & \multirow[b]{2}{*}{$\begin{array}{c}\text { Ontario } \\
\text { Population } \\
\text { Denominator }^{3}\end{array}$} & \multirow[b]{2}{*}{$\begin{array}{c}\text { Pts. Seen by a } \\
\text { Rheumatologist }{ }^{4}, \\
\text { n }(\%)\end{array}$} & \multicolumn{4}{|c|}{ Any Type of Patient Encounter ${ }^{1}$} & \multirow[b]{2}{*}{$\begin{array}{l}\text { Pts. Seen in } \\
\text { an Outpatient } \\
\text { Rheumatology } \\
\text { Clinic }^{7}, \mathrm{n}(\%)\end{array}$} & \multicolumn{3}{|c|}{ Outpatient Assessments Only ${ }^{2}$} & \multirow[b]{2}{*}{$\begin{array}{l}\text { New Pts. } \\
\text { among } \\
\text { Total } \\
\text { Outpatient } \\
\text { ssessments, \% }\end{array}$} \\
\hline & & & $\begin{array}{l}\text { Total } \\
\text { Pt. } \\
\text { Encounters }^{5}\end{array}$ & $\begin{array}{l}\text { New } \\
\text { Pts. }\end{array}$ & $\begin{array}{l}\text { New Pts. } \\
\text { among } \\
\text { Total Pts., \% } \\
\text { E }\end{array}$ & $\begin{array}{c}\text { New Pts. } \\
\text { among } \\
\text { Total } \\
\text { Encounters, \% }\end{array}$ & & $\begin{array}{c}\text { Total } \\
\text { Outpatient } \\
\text { Assessments }\end{array}$ & $\begin{array}{l}\text { New } \\
\text { Pts. }^{6}\end{array}$ & $\begin{array}{c}\text { New Pts. } \\
\text { among } \\
\text { Total Pts., \% } \\
\text { As }\end{array}$ & \\
\hline 2000 & $9,093,486$ & $245,486(2.7)$ & 561,452 & 145,686 & 59 & 26 & $172,864(1.9)$ & 382,074 & 89,426 & 52 & 23 \\
\hline 2001 & $9,241,575$ & $255,289(2.8)$ & 588,581 & 132,490 & 52 & 23 & $179,370(1.9)$ & 394,532 & 80,191 & 45 & 20 \\
\hline 2002 & $9,393,473$ & $263,139(2.8)$ & 599,278 & 126,811 & 48 & 21 & $182,111(1.9)$ & 400,879 & 75,081 & 41 & 19 \\
\hline 2003 & $9,527,281$ & $250,176(2.6)$ & 571,366 & 110,164 & 44 & 19 & $180,385(1.9)$ & 391,450 & 71,270 & 40 & 18 \\
\hline 2004 & $9,651,626$ & $258,751(2.7)$ & 599,821 & 105,906 & 41 & 18 & $185,891(1.9)$ & 398,768 & 68,146 & 37 & 17 \\
\hline 2005 & $9,788,275$ & $273,462(2.8)$ & 634,730 & 109,021 & 40 & 17 & $188,297(1.9)$ & 404,593 & 65,469 & 35 & 16 \\
\hline 2006 & $9,907,932$ & $286,309(2.9)$ & 663,302 & 111,136 & 39 & 17 & $195,043(2.0)$ & 417,241 & 66,509 & 34 & 16 \\
\hline 2007 & $10,125,042$ & $280,072(2.8)$ & 652,933 & 98,586 & 35 & 15 & $191,950(1.9)$ & 408,219 & 60,575 & 32 & 15 \\
\hline 2008 & $10,268,935$ & $282,959(2.8)$ & 667,337 & 95,510 & 34 & 14 & 196,687 (1.9) & 421,871 & 61,333 & 31 & 15 \\
\hline 2009 & $10,410,050$ & $284,158(2.7)$ & 685,195 & 94,123 & 33 & 14 & 201,649 (1.9) & 434,601 & 63,546 & 32 & 15 \\
\hline 2010 & $10,528,197$ & $285,103(2.7)$ & 693,667 & 92,435 & 32 & 13 & 202,618 (1.9) & 434,304 & 62,174 & 31 & 14 \\
\hline 2011 & $10,587,857$ & $288,422(2.7)$ & 725,807 & 89,393 & 31 & 12 & $212,479(2.0)$ & 460,309 & 65,151 & 31 & 14 \\
\hline 2012 & $10,716,779$ & 281,754 (2.6) & 698,384 & 85,853 & 31 & 12 & $219,466(2.1)$ & 447,380 & 67,648 & 31 & 15 \\
\hline 2013 & $10,881,690$ & $293,704(2.7)$ & 742,952 & 88,895 & 30 & 12 & $228,237(2.1)$ & 470,367 & 68,539 & 30 & 15 \\
\hline 2014 & $11,039,248$ & $292,948(2.7)$ & 746,300 & 86,793 & 30 & 12 & $229,026(2.1)$ & 473,788 & 65,214 & 29 & 14 \\
\hline 2015 & $11,103,150$ & $302,336(2.7)$ & 786,061 & 86,877 & 29 & 11 & $242,761(2.2)$ & 500,831 & 68,916 & 28 & 14 \\
\hline
\end{tabular}

${ }^{1}$ Any type of patient encounter includes inpatient, outpatient assessments, and non-face-to-face encounters. ${ }^{2}$ Outpatient assessments include only face-to-face patient assessments. ${ }^{3}$ Population denominator confined to residents aged 18 years and older. ${ }^{4}$ No. unique patients seen annually in an outpatient or inpatient setting with the \% reflecting the percentage of patients seen by rheumatologists for any type of rheumatology encounter and the denominator being the Ontario population. ${ }^{5}$ Total patient encounters include initial and repeat encounters. ${ }^{6}$ No. new patients seen by a rheumatologist. ${ }^{7} \%$ reflecting the proportion of patients seen by a rheumatologist in an outpatient setting and the denominator being the Ontario population. ${ }^{8}$ Total outpatient assessments include initial and repeat encounters. Pt(s).: patient(s).

Personal non-commercial use only. The Journal of Rheumatology Copyright @ 2020. All rights reserved. 


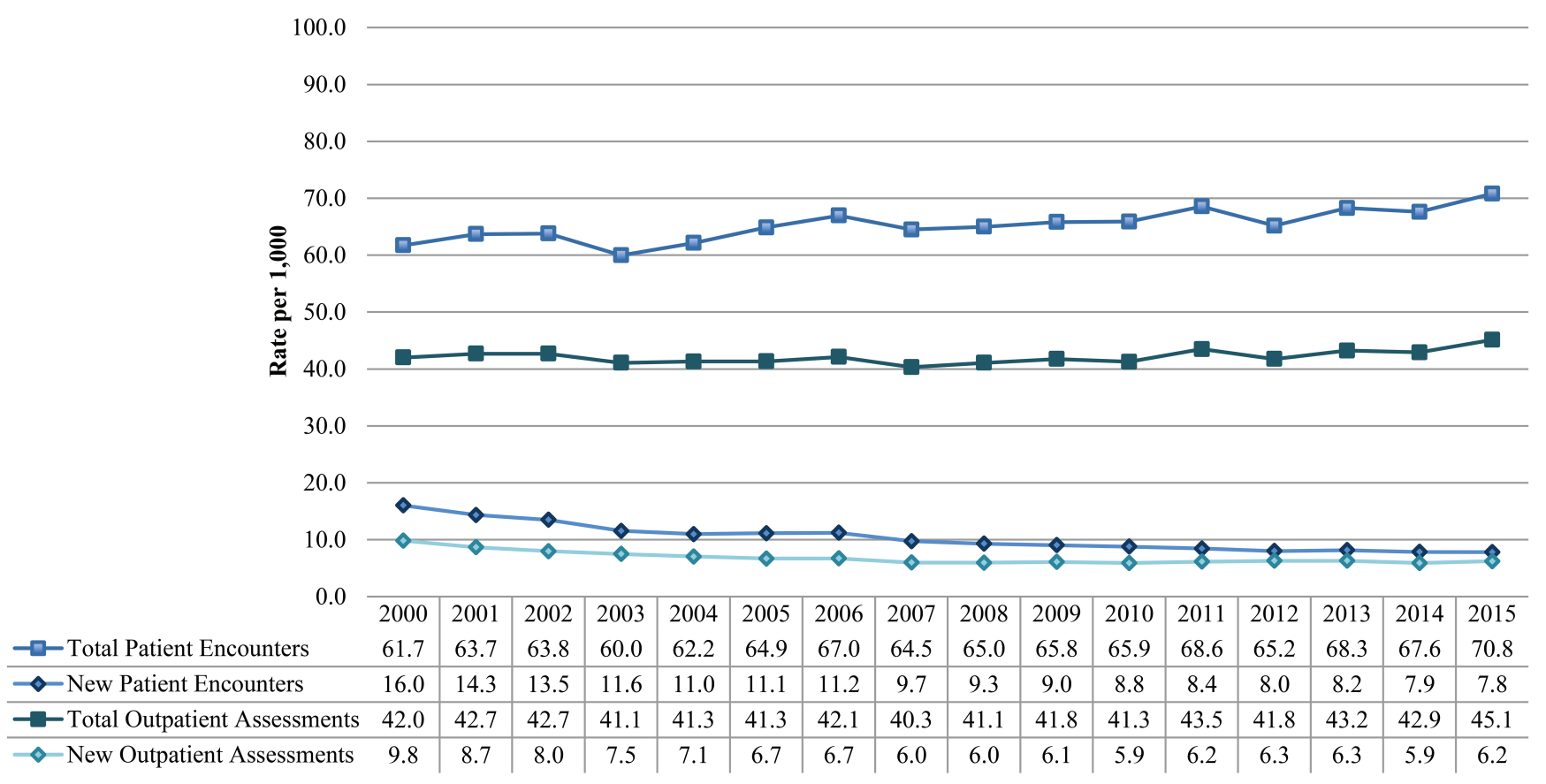

Figure 1. Rates of encounters with rheumatologists, per 1000 population.

tions increased over time whereas nonsystemic inflammatory conditions rates decreased.

Provider-level activity. In 2000, there were 146 adult rheumatologists in Ontario (57 of whom worked $<1$ clinical FTE; 31 worked as at least 1 clinical FTE; and 58 as $>1$ clinical FTE). This increased to 194 rheumatologists ( $74<1$ FTE; 41 FTE; $79>1$ FTE) in 2015, corresponding to an overall provincial per capita supply of 0.8 FTE per 75,000 (Supplementary Figure 2, available with the online version of this article). As of 2000, the total rheumatology workforce was predominantly male $(66 \%)$, with a mean (SD) age of 48.5 (10.6) years, and $16 \%$ of rheumatologists were aged 60 years and older. By $2015,51 \%$ of the workforce was male, a mean (SD) age of 51.7 (11.6) years, and 26\% were over 60 years of age. More male rheumatologists were consistently identified as $>1$ clinical FTE each year (Supplementary Figure 2), with male rheumatologists providing more patient assessments than female rheumatologists, across all age groups (data not shown).

The annual median (interquartile range) number of days in which rheumatologists had patient encounters decreased from 231 (193-257) days in 2000 to 205.5 (159-253) days in 2015 (Table 3). When we considered assessments only, rheumatologists had even fewer days of clinical activity (180 days/yr in 2015). The percentage of rheumatologists with patient encounters on at least 209 days/year (an alternative FTE benchmark) showed a downward trend over time. When we restricted the analysis to patient assessments and varied the criteria of the number of patient assessments within each day, a similar downward trend over time was observed (Supplementary Figure 3, available with the online version of this article).

Within each FTE category, annual rheumatology practice volumes and practice sizes also decreased over time (Table 4). In 2015, the average rheumatologist practicing as $<1$ clinical FTE saw 670 patients, rheumatologists practicing as 1 clinical FTE saw 1219 patients, and those providing more clinical service ( $>1$ clinical FTE) saw 2050 patients. Monthly and daily practice volumes are provided in Supplementary Table 4 (available with the online version of this article). The median numbers of new patients seen annually and monthly significantly decreased over time within each FTE classification (Supplementary Table 5).

\section{DISCUSSION}

We conducted a large population-based study of all contacts with rheumatologists in a universal healthcare system and quantified changes in clinical activity of rheumatologists over a 15-year period. Our study reveals that while the crude volume of rheumatology contacts increased annually over time, the proportion of the population under rheumatology care remained constant and the adjusted total encounter and assessment rates remained relatively stable over time. The annual new patient consultation rate significantly declined over time as fewer new patients were seen annually. During the study period, Ontario held a constant ratio of 0.8 clinical FTE rheumatologists per 75,000 population (Supplementary Figure 2, available with the online version of this article). Personal non-commercial use only. The Journal of Rheumatology Copyright @ 2020 . All rights reserved. 


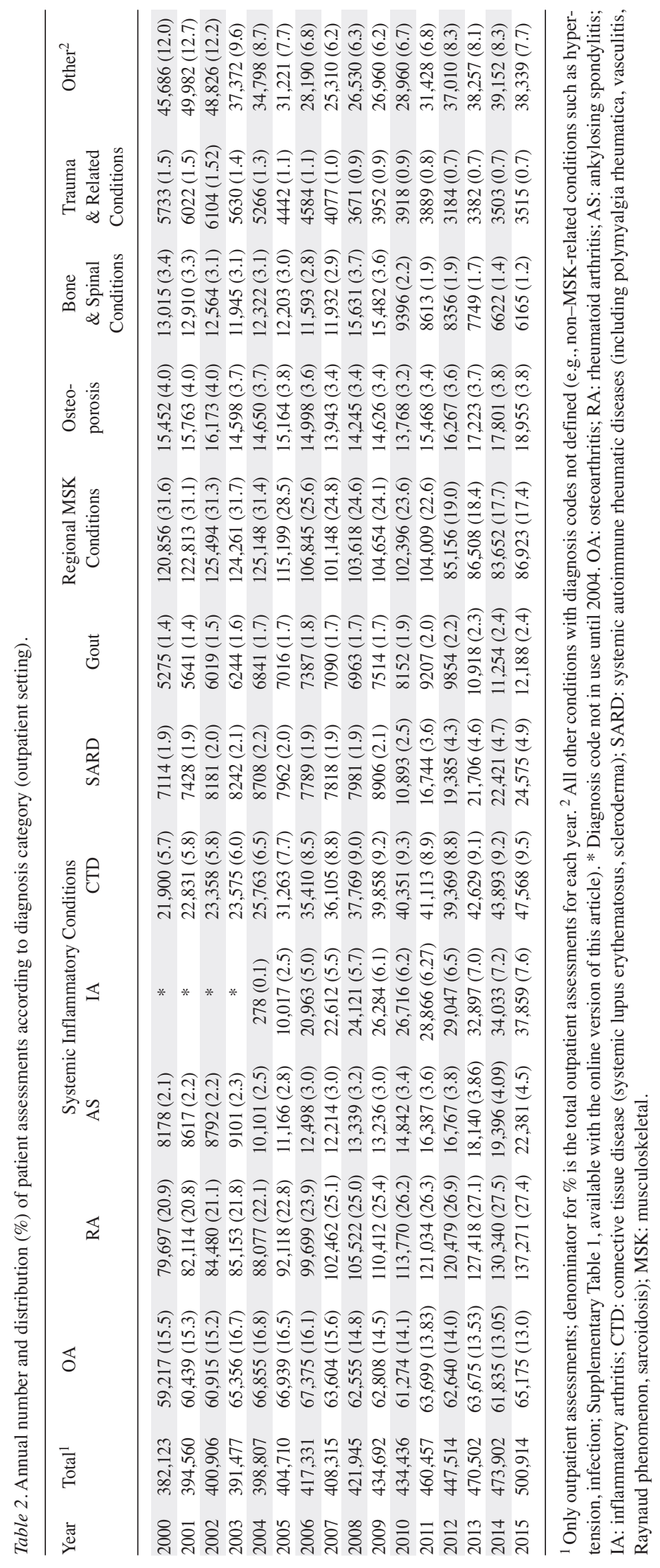

Personal non-commercial use only. The Journal of Rheumatology Copyright (c) 2020. All rights reserved. 
Table 3. Annual number of days rheumatologists provided clinical activity.

\begin{tabular}{|c|c|c|c|c|}
\hline \multirow[b]{2}{*}{ Year } & \multicolumn{2}{|c|}{ Any Type of Patient Encounter ${ }^{1}$} & \multicolumn{2}{|c|}{ Patient Assessments Only ${ }^{2}$} \\
\hline & $\begin{array}{l}\text { Annual No. Days with Any } \\
\text { Type of Patient Encounter, } \\
\text { Median (IQR) }\end{array}$ & $\begin{array}{l}\text { Rheumatologists with At Least } \\
3 \text { Patient Encounters on } \\
\text { At Least } 209 \text { Days per Year }{ }^{3}, \mathrm{n}(\%)\end{array}$ & $\begin{array}{l}\text { Annual No. Days with } \\
\text { Patient Assessments, } \\
\text { Median (IQR) }\end{array}$ & $\begin{array}{l}\text { Rheumatologists with At Least } \\
3 \text { Patient Assessments on } \\
\text { At Least } 209 \text { Days per Year }{ }^{3,4}, \mathrm{n}(\%)\end{array}$ \\
\hline 2000 & $231(193-257)$ & $78(53)$ & $220(178-243)$ & $67(46)$ \\
\hline 2001 & $227(191.5-255.5)$ & $75(49)$ & $216(169-237)$ & $61(40)$ \\
\hline 2002 & $226(192-255)$ & $77(50)$ & $210.5(168-238)$ & $63(41)$ \\
\hline 2003 & $224.5(187-251)$ & $78(53)$ & $209(165-233)$ & $66(45)$ \\
\hline 2004 & $222(182-250)$ & $77(52)$ & $203.5(160.5-229)$ & $62(42)$ \\
\hline 2005 & $222(175-251)$ & $73(48)$ & $202(151-234)$ & $57(38)$ \\
\hline 2006 & $218(171-251)$ & $72(47)$ & $195.5(157-231)$ & $53(34)$ \\
\hline 2007 & $210(166-248)$ & $69(45)$ & $189(143-224)$ & $49(32)$ \\
\hline 2008 & 209.5 (164-246.5) & $67(43)$ & $189.5(150-223)$ & $46(30)$ \\
\hline 2009 & $206.5(161.5-244)$ & $64(40)$ & $182.5(142.5-218)$ & $43(27)$ \\
\hline 2010 & $206(161-244)$ & $68(42)$ & $182(145-214)$ & $42(26)$ \\
\hline 2011 & $207.5(155-241.5)$ & $73(42)$ & $178(134-219)$ & $46(26)$ \\
\hline 2012 & $207(156-243)$ & $63(36)$ & 175 (137-209) & $34(19)$ \\
\hline 2013 & $208(157-250)$ & $72(39)$ & $176(138-213)$ & $40(22)$ \\
\hline 2014 & $204(165-248)$ & $71(39)$ & $177(137-212)$ & $42(23)$ \\
\hline 2015 & $205.5(159-253)$ & $75(39)$ & $180(137-208)$ & $43(22)$ \\
\hline
\end{tabular}

${ }^{1}$ Any type of patient encounter includes inpatient, outpatient assessments, and non-face-to-face encounters. ${ }^{2}$ Patient assessments include only face-to-face patient assessments. ${ }^{3}$ Denominator is the total no. active rheumatologists within each year (see Supplementary Figure 1 for denominators, available with the online version of this article). ${ }^{4}$ See Supplementary Figure 3, comparing different criteria of the no. daily patient assessments. IQR: interquartile range.

Table 4. Median (IQR) annual rheumatology practice volumes (no. patient encounters per year) and practice sizes (no. patients seen per year) by clinical FTE classification.

\begin{tabular}{lcccccc}
\hline \multirow{2}{*}{ Year } & \multicolumn{2}{c}{$<$ 1 Clinical FTE } & \multicolumn{2}{c}{ 1 Clinical FTE } & & \multicolumn{2}{c}{ C Clinical FTE $^{3}$} \\
& Practice Volume & Practice Size & Practice Volume & Practice Size & Practice Volume & Practice Size \\
\hline 2000 & $1730(879-2494)$ & $796(411-1108)$ & $3467(2842-4727)$ & $1418(1160-1922)$ & $5752(4586-6733)$ & $2396(1709-3320)$ \\
2001 & $1768(933-2478)$ & $789(420-1081)$ & $3381(2837-4327)$ & $1398(1207-1765)$ & $5763(4791-6721)$ & $2507(2064-3388)$ \\
2002 & $1804(797-2658)$ & $773(439-1034)$ & $3487(2922-3774)$ & $1416(1217-1712)$ & $5552(4743-7098)$ & $2385(1900-3374)$ \\
2003 & $1697(827-2323)$ & $746(449-1031)$ & $3365(2868-4228)$ & $1375(1238-1678)$ & $5632(4536-7002)$ & $2432(1921-3245)$ \\
2004 & $1823(867-2513)$ & $814(404-1071)$ & $3734(3255-4410)$ & $1497(1330-1843)$ & $5820(4782-7703)$ & $2367(1904-3369)$ \\
2005 & $1772(600-2459)$ & $759(380-1033)$ & $3625(2957-4491)$ & $1456(1275-1580)$ & $5961(4955-8159)$ & $2380(1923-3307)$ \\
2006 & $1866(743-2371)$ & $779(384-1015)$ & $3584(2996-4787)$ & $1373(1254-1521)$ & $5971(4719-8416)$ & $2406(1912-3617)$ \\
2007 & $1722(730-2282)$ & $737(380-1016)$ & $3561(2934-4559)$ & $1347(1174-1579)$ & $5776(4603-8561)$ & $2476(1830-3311)$ \\
2008 & $1738(676-2283)$ & $815(346-1019)$ & $3405(2905-4035)$ & $1335(1182-1504)$ & $5911(4466-8496)$ & $2199(1808-3164)$ \\
2009 & $1662(679-2259)$ & $816(387-951)$ & $3575(2970-4195)$ & $1324(1116-1561)$ & $5780(4471-8358)$ & $2310(1801-3094)$ \\
2010 & $1745(737-2323)$ & $798(376-975)$ & $3459(2987-4370)$ & $1325(1147-1554)$ & $5524(4508-8289)$ & $2242(1852-3082)$ \\
2011 & $1536(677-2004)$ & $663(328-913)$ & $3485(2883-4090)$ & $1229(1080-1581)$ & $5533(4416-7743)$ & $2092(1735-2864)$ \\
2012 & $1525(892-2136)$ & $713(427-887)$ & $3316(2996-3900)$ & $1170(1061-1455)$ & $5357(4362-7704)$ & $2101(1750-2700)$ \\
2013 & $1598(859-2187)$ & $687(349-869)$ & $3340(2873-3832)$ & $1196(985-1334)$ & $5215(4260-7525)$ & $1988(1702-2701)$ \\
2014 & $1739(915-2256)$ & $737(420-869)$ & $3365(2863-3868)$ & $1230(1030-1406)$ & $5277(4342-7698)$ & $1940(1655-2518)$ \\
2015 & $1492(663-2234)$ & $670(409-890)$ & $3315(2891-3763)$ & $1219(1022-1337)$ & $5547(4553-7658)$ & $2050(1756-2563)$ \\
& & & & & &
\end{tabular}

${ }^{1}$ Among rheumatologists identified as those with $<40$ th percentile of total billings. ${ }^{2}$ Among rheumatologists identified as those in the $40-60$ th percentile of total billings. ${ }^{3}$ Among rheumatologists identified as those $>60$ th percentile of total billings (thus providing more clinical service). FTE: full-time equivalent; IQR: interquartile range.

Without an increase in the per capita supply of rheumatologists, the proportional increase of rheumatology encounters occurring with established patients may also be limiting access for new consultations and increasing wait times. We also observed a shift in patient case mix over time, with rheumatologists seeing more systemic inflammatory conditions. Our practice-level findings of the decline of clinical activity of rheumatologists over time provide important information for rheumatology workforce planning.

Across Canada, including Ontario, there is a lower per capita supply of rheumatologists than in the United States ${ }^{4}$. A comprehensive US workforce study reported a provider/population ratio of 3.1 per 100,000 in the Northeast to 1.3 per 100,000 in the Southwest in $2015^{3}$. Within the

Personal non-commercial use only. The Journal of Rheumatology Copyright $\subset$ 2020 . All rights reserved. 
United States, there was an estimated deficit of 700 FTE rheumatologists in 2015 and by 2030, the projected demand will exceed the supply by 4133 clinical $\mathrm{FTE}^{3}$. Moreover, studies have projected a significant increase in patient demand for rheumatologists 3,16 .

In Canada, there were about 400 rheumatologists as of $2015^{4,17}$, with an estimated deficit of 200 rheumatologists ${ }^{4}$. The current shortage is further compounded by one-third of the workforce nearing retirement ${ }^{4}$, which is raising concerns over whether there are sufficient numbers of positions in rheumatology training programs to replace rheumatologists who retire, in addition to expanding workforce requirements needed to meet the increasing demands for rheumatology care associated with the growing and aging population.

We also observed changes in clinical activity over time, with a decreasing number of patient encounters per rheumatologist per year and fewer days providing clinical care annually. Previous studies have suggested that the clinical activity of rheumatologists may be declining ${ }^{3,16}$. Evidence from rheumatologist surveys suggests that the average number of visits provided annually by a rheumatologist varies depending on the age and sex of the rheumatologist ${ }^{16,18}$, with the average male rheumatologist providing about 35 percent more visits annually than the average female $^{16}$. A recent Canadian survey reported that although female rheumatologists saw fewer patients, they worked equivalent hours compared to their male counterparts ${ }^{18}$. Our study also revealed that more male rheumatologists practice as clinical FTE compared to female rheumatologists (Supplementary Figure 2, available with the online version of this article). This has implications for planning and managing services. With the increasing feminization of rheumatology workforces, longer consultations by female rheumatologists will limit the number of consultations they can provide to the population. In addition, generational differences (including millennials who place more emphasis on the value of both leisure time and earnings ${ }^{19}$ ), and the increasing age of the workforce is likely to influence workforce capacity, as has been demonstrated in other specialties $20,21,22$. Additional factors affecting rheumatology clinical activity may include clinic saturation, increasing care complexity, different practice models of care (e.g., differential access to allied health providers), greater demands for CME, and research activity. More research of the effects of these factors on rheumatology workforces is warranted.

Our study also suggests that Ontario rheumatologists may be managing this imbalance of supply and demand by changing how they practice and following more inflammatory conditions (which require ongoing followup care). Additionally, the volume of rheumatology inpatient encounters declined over time, which is likely reflective of the declining number of rheumatologists with hospital affiliations (data not shown), resulting in more outpatient care, as opposed to fewer hospitalizations for patients with RMD.
We used population-based data from a large single-payer healthcare system, which has the strength of being relatively complete for rheumatology billing claims and population coverage. However, we acknowledge some potential limitations of our study. First, we observed an increase in the crude total patient encounter rate but not the outpatient assessment rate over time; therefore, the total patient encounter rate needs to be interpreted with caution, because it may reflect an increase in the availability of additional fee-for-service billing fee codes over time (such as billing claims to review laboratory test reports). Yet the total patient encounter rate (which records non-face-to-face interactions) may better determine the totality of patient interactions. A previous time and motion study found that for every office hour spent on direct clinical face time with patients, physicians spent nearly an additional 2 hours on electronic health records and desk work $^{23}$. Thus, while health administrative data represent an advantage of this study, we are unable to assess the totality of physician activity (e.g., time spent with patients, and in completion of forms and review of health records before and after direct patient interactions).

Another potential caveat is that our data allowed us only to assess patients who were seen by rheumatologists, and not all patients are referred to rheumatologists. In our study, we observed fewer patients being seen with nonsystemic inflammatory conditions (e.g., OA and regional self-limiting MSK conditions) over time. This trend may be a reflection of rheumatologists declining these types of referrals in more recent years and instead seeing more patients with systemic inflammatory conditions, and/or fewer referrals to rheumatologists for these noninflammatory conditions. Indeed, in a study of 2430 patients referred to a rheumatologist in Ontario, $17 \%$ did not result in a rheumatology consultation ${ }^{10}$.

Our data are also limited in that physician service claims only require 1 diagnosis code per patient encounter, limiting what is reported for patients who have multiple health problems. Diagnosis codes assigned at patient assessments also may not be accurate, or could be used when a disease is being "ruled out." Temporal variation in billing code practices during the study period could influence our results. Changes over time in billing could be attributable to true changes in case mix, improvements in coding (because of provider education and in degree of detail in codes or their definitions, or the use of electronic medical records), or code creep (physicians using codes associated with higher reimbursement $)^{24}$. In Ontario, there are additional premiums associated with systemic inflammatory conditions (where patients require more complex care). However, the increased prevalence of systemic inflammatory conditions within rheumatology practices over time likely reflects that these patients are remaining under rheumatology care rather than physicians attempting to increase their reimbursement. Higher reimbursement is provided because these patients require more complex care (and subsequently more physician

Personal non-commercial use only. The Journal of Rheumatology Copyright $\subset$ 2020. All rights reserved. 
time with the patient). While it is possible that changes to the fee code structure have influenced the increase in followup of patients with RA, it is also possible that the increase in followup of patients with RA (and other systemic inflammatory conditions) is aligned with best practice care ${ }^{25}$. A significantly larger proportion of patients with systemic inflammatory conditions had followup encounters with their rheumatologist compared with noninflammatory conditions. In 2000 , only $58 \%$ of patients billed for OA on their initial rheumatology visit had a subsequent rheumatology visit within 12 months. The percentage declined steadily over time, with only $36 \%$ of patients with OA having a followup visit in 2014. Moreover, we found a decline in the percentage of total new patients being accepted into rheumatology practices over time, which likely reflects that practices are becoming saturated with patients requiring continuous care (such as those with systemic inflammatory conditions) and limiting the availability of new consultations.

Currently, changes to rheumatology wait times over time are unknown; however, wait times exceed established benchmarks ${ }^{10}$. An alternative explanation to the reduction in new consultations is that some patients (such as those with regional MSK conditions) are being referred to other care providers. However, in our study, rheumatology practice sizes and volumes exceed those of family physicians ${ }^{26,27}$, and thus it is likely that clinic saturation is playing a role in reducing the availability of rheumatologists to see new patients.

Finally, there is no perfect methodology or consensus to define a clinical FTE rheumatologist. For physicians in general, hours worked are unknown, apart from self-reported data, which may be unreliable. Defining FTE from fee-for-service billings is commonly used as a proxy for time worked. The methodology we used (which determined total fee-for-service billings for each rheumatologist annually and those below the 40th percentile defined as < 1 FTE) was chosen for consistency through time because this methodology removes the effects of different fee levels on physician income. Simulations of alternative percentiles have shown that the FTE counts are relatively insensitive to different benchmark ranges (e.g., 60th vs 70th percentile to define high volume providers $)^{15}$. However, we acknowledge that any definition of FTE is arbitrary, the methodology may be imperfect, and thus we present an alternative definition (annual number of days providing clinical care).

Our findings provide novel insights for rheumatology workforce planning. Access to rheumatologists in Ontario has not increased over time. The significant decline in new patient consultation rates over time helps illustrate the growing supply-demand mismatch in rheumatology care. An increasing fraction of rheumatology encounters are with established patients, which may be limiting access for new consultations and increasing wait times. We observed changes in the volumes of clinical activity and the types of patients being seen by rheumatologists. Our findings have important implications for people with RMD, care providers, and health policy makers.

\section{ONLINE SUPPLEMENT}

Supplementary material accompanies the online version of this article.

\section{ACKNOWLEDGMENT}

The authors thank Sue Schultz and Alex Kopp for their expertise.

\section{REFERENCES}

1. Briggs AM, Cross MJ, Hoy DG, Sanchez-Riera L, Blyth FM, Woolf $\mathrm{AD}$, et al. Musculoskeletal health conditions represent a global threat to healthy aging: a report for the 2015 World Health Organization World Report on Ageing and Health. Gerontologist 2016;56 Suppl 2:S243-55.

2. Al Maini M, Adelowo F, Al Saleh J, Al Weshahi Y, Burmester GR, Cutolo M, et al. The global challenges and opportunities in the practice of rheumatology: white paper by the world forum on rheumatic and musculoskeletal diseases. Clin Rheumatol 2015;34:819-29.

3. Battafarano DF, Ditmyer M, Bolster MB, Fitzgerald JD, Deal C, Bass AR, et al. 2015 American College of Rheumatology workforce study: supply and demand projections of adult rheumatology workforce, 2015-2030. Arthritis Care Res 2018;70:617-26.

4. Barber CE, Jewett L, Badley EM, Lacaille D, Cividino A, Ahluwalia $\mathrm{V}$, et al. Stand up and be counted: measuring and mapping the rheumatology workforce in Canada. J Rheumatol 2017;44:248-57.

5. Widdifield J, Paterson JM, Bernatsky S, Tu K, Thorne JC, Ahluwalia $\mathrm{V}$, et al. The rising burden of rheumatoid arthritis surpasses rheumatology supply in Ontario. Can J Public Health 2013;104:e450-5.

6. Bolster MB, Bass AR, Hausmann JS, Deal C, Ditmyer M, Greene KL, et al. 2015 American College of Rheumatology workforce study: the role of graduate medical education in adult rheumatology. Arthritis Rheumatol 2018;70:817-25.

7. Liddy C, Arbab-Tafti S, Moroz I, Keely E. Primary care physician referral patterns in Ontario, Canada: a descriptive analysis of self-reported referral data. BMC Fam Pract 2017;18:81.

8. Jaakkimainen L, Glazier R, Barnsley J, Salkeld E, Lu H, Tu K. Waiting to see the specialist: patient and provider characteristics of wait times from primary to specialty care. BMC Fam Pract 2014;15:16.

9. Shadd J, Ryan BL, Maddocks H, Thind A. Patterns of referral in a Canadian primary care electronic health record database: retrospective cross-sectional analysis. Inform Prim Care 2011;19:217-23.

10. Widdifield J, Bernatsky S, Thorne JC, Bombardier C, Jaakkimainen $\mathrm{RL}$, Wing L, et al. Wait times to rheumatology care for patients with rheumatic diseases: a data linkage study of primary care electronic medical records and administrative data. CMAJ Open 2016; 4:E205-12.

11. Widdifield J, Tu K, Carter Thorne J, Bombardier C, Michael Paterson J, Liisa Jaakkimainen R, et al. Patterns of care among patients referred to rheumatologists in Ontario, Canada. Arthritis Care Res 2017;69:104-14.

12. Statistics Canada. Canada's ethnocultural portrait: the changing mosaic. Archived. [Internet. Accessed October 24, 2019.] Available from: https://www150.statcan.gc.ca

13. Statistics Canada. Population estimates on July 1st, by age and sex. [Internet. Accessed October 24, 2019.] Available from: https://doi.org/10.25318/1710000501-eng

Personal non-commercial use only. The Journal of Rheumatology Copyright $\subset$ 2020. All rights reserved. 
14. Ontario Ministry of Health and Long-Term Care. OHIP schedule of benefits and fees. [Internet. Accessed October 8, 2019.] Available from: health.gov.on.ca/en/pro/programs/ohip/sob

15. Canadian Institute for Health Information. National physician database data release, 2015-2016: methodological notes. [Internet. Accessed October 8, 2019.] Available from: www.cihi.ca/sites/ default/files/document/npdb_data_release_methodology_notes_ phys2016_en.pdf

16. Deal CL, Hooker R, Harrington T, Birnbaum N, Hogan P, Bouchery E, et al. The United States rheumatology workforce: Supply and demand, 2005-2025. Arthritis Rheum 2007;56:722-9.

17. National Physician Survey. 2014 results for internal medicine subspecialists by specialty. [Internet. Accessed October 8, 2019.] Available from: nationalphysiciansurvey.ca/result/ 2014-results-internal-medicine-subspecialists-specialty

18. Barber CE, Nasr M, Barnabe C, Badley EM, Lacaille D, Pope J, et al. Planning for the rheumatologist workforce: factors associated with work hours and volumes. J Clin Rheumatol 2019;25:142-6.

19. Jovic E, Wallace JE, Lemaire J. The generation and gender shifts in medicine: an exploratory survey of internal medicine physicians. BMC Health Serv Res 2006;6:55.

20. Sarma S, Thind A, Chu MK. Do new cohorts of family physicians work less compared to their older predecessors? The evidence from Canada. Soc Sci Med 2011;72:2049-58.
21. Hedden L, Barer ML, Cardiff K, McGrail KM, Law MR, Bourgeault IL. The implications of the feminization of the primary care physician workforce on service supply: a systematic review. Hum Resour Health 2014;12:32.

22. Pannor Silver M, Easty LK. Planning for retirement from medicine: a mixed-methods study. CMAJ Open 2017;5:E123-9.

23. Sinsky C, Colligan L, Li L, Prgomet M, Reynolds S, Goeders L, et al. Allocation of physician time in ambulatory practice: a time and motion study in 4 specialties. Ann Intern Med 2016;165:753-60.

24. Seiber EE. Physician code creep: evidence in medicaid and state employee health insurance billing. Health Care Financ Rev 2007;28:83-93.

25. Bykerk VP, Akhavan P, Hazlewood GS, Schieir O, Dooley A, Haraoui B, et al; Canadian Rheumatology Association. Canadian Rheumatology Association recommendations for pharmacological management of rheumatoid arthritis with traditional and biologic disease-modifying antirheumatic drugs. J Rheumatol 2012; 39:1559-82.

26. Raffoul M, Moore M, Kamerow D, Bazemore A. A primary care panel size of 2500 is neither accurate nor reasonable. J Am Board Fam Med 2016;29:496-9.

27. Ministry of Health and Long-Term Care. Funding alternatives for family physicians. [Internet. Accessed October 8, 2019.] Available from: www.auditor.on.ca/en/content/annualreports/arreports/ en11/306en11.pdf 